\title{
Atomic scale modeling of structural phase transformations in AlCrFeMnMo high-entropy alloys during thermal treatments
}

\author{
W. Sekkal*, R. Besson*, A. Legris \\ Univ. Lille, CNRS, INRAE, Centrale Lille, UMR 8207 - UMET - Unité Matériaux et Transformations, F-59000 Lille, France
}

\section{A R T ICLE INFO}

\section{Article history:}

Received 29 March 2021

Received in revised form 21 April 2021

Accepted 24 April 2021

Available online $\mathrm{xxx}$

\begin{abstract}
A B S T R A C T
The objective of this work is to provide an overview of the evolution of phase transformations in AlCrFeMnMo high-entropy alloys (HEAs), a recently proposed family showing great promises in metallurgical industry for coating applications. We perform Monte-Carlo simulations based on ab initio energy parameters, to analyze the order/disorder transition and the phase separation process from the high-temperature A2 single phase. The reliability of the atomic-scale approach, already known to work on AlCoCrFeNi, is first confirmed on $\mathrm{AlCrFeMnNi}$, the presence of A2-(Cr,Fe, Mn) and B2-(Al,Ni) being in good agreement with previous experimental studies. However, simulations of equimolar AlCrFeMnMo show that the A2 solid solution should transform into two B2 phases at ambient temperature, instead of a couple of A2 phases observed experimentally. The reasons for this discrepancy are discussed. Increasing iron and decreasing molybdenum contents in AlCrFeMnMo favors ordering for chromium and phase change for aluminum, without changing the conclusion on A2 / B2 competition previously drawn from the equimolar case.
\end{abstract}

(C) 2021

\section{Introduction}

Since their discovery, high-entropy alloys (HEAs) have attracted great research interest because of their wide potential in structural and functional applications [1]. They are complex metallic solid solutions containing at least five metallic elements in equiatomic or nearly equiatomic ratios $[2,3]$. HEAs are only 17 years old and have already been shown to possess exceptional structural and mechanical properties, exceeding those of conventional alloys [4-6]. A remarkable number of research works on HEAs have been produced in the last years, most of them being experimental studies [7-9]. However, modeling and simulations of HEAs are currently paid increasing attention, as they provide powerful approaches complementing experiments and serving as predictive tools [10-16]. HEAs have high configurational entropy, which tends to stabilize the solid solution formation [17]. Therefore, thermodynamic stability is perhaps the most important feature to elucidate, when attempting to predict the expected phases during the elaboration processes of HEAs. In earlier studies, a so-called "parametric approach" has often been employed for a quick screening of the HEA composition space, in order to identify domains prone to solid solution

\footnotetext{
* Corresponding authors.

E-mail addresses: wassila.sekkal@univ-lille.fr (W. Sekkal); remy.besson@univ-lille.fr (R. Besson)
}

stability $[12,17]$. This approach included a wide number of parameters to guide composition optimization and phase selection, such as mixing entropy [18], atomic size difference [19], ratio parameter [20], mixing enthalpy [19], geometrical analysis [21], and valence electron concentration [22]. Although it proved to be useful in practice to help in HEA selection, the parametric approach inherently suffers from a lack of justification and insight into the physical mechanisms underlying HEA formation and stability. To bridge this gap, atomic-scale approaches are especially appealing, since they naturally provide information on interspecies bonding properties and their major consequences on phase order and stability in HEAs. In particular, a better insight into short- and long-range order and single- or multi-phasing could open interesting perspectives of comparison with advanced atomic-scale experimental techniques. However, as often in such theoretical studies the major difficulty lies in the selection of a proper energy model able to deal with the multicomponent and concentrated nature of HEAs. In a noticeable attempt to circumvent this issue, Troparevsky et al. [23] performed massive ab initio calculations of formation enthalpies for many binary compounds. They deduced from these extended data a large set of nearest-neighbor pair interaction energy parameters between $\sim 30$ chemical elements, thus offering an interesting ground to investigate many HEAs at the atomic scale. More recently [24], machine-learning studies have been performed on a large dataset $(1,252$ elements), leading to propose a new thermodynamics-based empirical rule to predict physical properties of HEAs, such as molar vol- 
ume, bulk modulus and melting temperature. This rule may help to predict reliably (93\%) the solid solution formation of multicomponent alloys. Within this rapidly growing field of experimental and theoretical researches on HEAs, researchers are dealing now with the emergence of non-equimolar multiphase HEAs [25], including secondary phases rather than a single phase in traditional HEAs. This increasing complexity makes it especially important to thoroughly investigate the theoretical and simulation tools available and possibly conceivable, in order to explore efficiently the immense compositional space spanned by HEAs, a task that cannot be achieved by experimental efforts alone.

In the present paper, we propose to study the AlCrFeMnMo system, which provides a novel family of promising HEA candidates for coating applications. Very few experimental investigations have been done on this quinary system up to now, the only work being due to Stasiak et al. [26], who studied AlCrFeMnMo by mechanical alloying. Their experiments allowed identifying two body-centered cubic (bcc) phases $\left(\mathrm{A} 2^{\prime}+\mathrm{A} 2^{\prime \prime}\right)$ mainly enriched in Mo and Cr respectively. On the other hand, Senkov et al. [27], from a CALPHAD analysis on this quinary system, concluded that two phases, i.e. the A2 bcc solid solution and an $\mathrm{AlMo}_{3}$ ordered compound, should be stable in a temperature range from $600{ }^{\circ} \mathrm{C}$ to $1040{ }^{\circ} \mathrm{C}$. These earlier studies evidence clear disagreements between experiments and theoretical calculations on AlCrFeMnMo, which suggests that further investigations should be done on this particular HEA family.

In this context, the present work is devoted to atomic-scale simulations of the phase transformations in equimolar AlCrFeMnMo and "optimized" ${ }^{26} \mathrm{Al}_{22} \mathrm{Cr}_{22} \mathrm{Fe}_{29} \mathrm{Mn}_{20} \mathrm{Mo}_{7}$ predicted to occur at cooling from nearly melting $\left(1600{ }^{\circ} \mathrm{C}\right)$ to $0{ }^{\circ} \mathrm{C}$ temperature. Using the aforementioned configuration nearest-neighbor pair energy model [23,28], and Monte-Carlo simulations, we mainly aim at predicting the equilibrium phases expected in this couple of AlCrFeMnMo alloys. Nevertheless, as this approach was used earlier [29] on AlCoCrFeNi and gave consistent results with experimental findings, this suggests reaching AlCrFeMnMo gradually, through an intermediate step provided by equimolar $\mathrm{AlCrFeMnNi}$, a system already studied experimentally by Zhang et al. [30], but never investigated with atomic-scale simulations. The overall scheme of our study can therefore be seen as following the AlCoCrFeNi $\rightarrow \mathrm{AlCrFeMnNi} \rightarrow \mathrm{AlCrFeMnMo}$ alloy sequence.

Our main objective in this work is to analyze (i) the order/disorder transition in the high-temperature A2 disordered solid solution, and (ii) the phase separation in the single-phase system, as well as the effect of composition and temperature on these trends.

\section{Methodology}

In the approach used here, the interatomic interactions between elements $i$ and $j$ are limited to nearest neighbors [28], the binary interatomic energies $v_{i j}$ being obtained from the binary formation enthalpies of the most stable phases, derived from ab initio calculations [23] (Table 1). In this framework, the alloy enthalpy is expressed in terms of long-range order parameters $\eta_{i}$ as follows:

Table 1

Nearest-neighbor interaction energies $(\mathrm{kJ} / \mathrm{mol})$ (data obtained from earlier first-principles calculations [23]). See also Fig. 11.

\begin{tabular}{lllllll}
\hline Element & Al & Cr & Fe & Mn & Mo & Ni \\
\hline Al & 0 & -1.66 & -4.44 & -3.35 & -2.02 & -8.15 \\
Cr & -1.66 & 0 & -0.10 & -1.32 & 0.50 & -0.36 \\
Fe & -4.44 & -0.10 & 0 & 0.11 & -5.83 & -1.17 \\
Mn & -3.35 & -1.32 & 0.11 & 0 & -1.66 & -1.39 \\
Mo & -2.02 & 0.50 & -5.82 & -1.66 & 0 & -1.20 \\
Ni & -8.15 & -0.36 & -1.17 & -1.39 & -1.20 & 0 \\
\hline
\end{tabular}

$\Delta H_{\text {mix }}=4 \sum_{i=1, j \neq i}^{n} v_{i j} x_{i}\left(1+\eta_{i}\right) x_{j}\left(1-\eta_{i}\right)$

where $n$ is the number of chemical elements, $R$ is the gas constant and $T$ is the temperature.

In the $\mathrm{AlCrFeMnNi}$ system, the most noticeable trend concerns the low absolute values for Cr-Fe-Mn-Ni energy parameters, which means weak interactions between these four elements, while aluminum strongly interacts with nickel. In AlCrFeMnMo (Ni replaced with Mo), we also note weak interactions for $\mathrm{Cr}-\mathrm{Fe}-\mathrm{Mn}-\mathrm{Mo}$, except for Fe-Mo $(-5.83 \mathrm{~kJ} / \mathrm{mol})$. Positive $\mathrm{Cr}$-Mo and Fe-Mn interactions are also noticed, hence a separation tendency expected for each of these couples of elements. Moreover, interactions of $\mathrm{Al}$ with $\mathrm{Cr}, \mathrm{Fe}, \mathrm{Mn}$ and Mo are attractive.

AlCrFeMnMo HEAs primarily involve more or less ordered phases formed on the bcc structure with a lattice parameter of $3.16 \AA$ for the equimolar alloy (note that, as the $\nu_{\mathrm{ij}}$ do not depend on interatomic distances, the value of this parameter does not affect the results). In the present rigid-lattice simulations of these systems, we used a 27,648-site supercell constituted of $24 \times 24 \times 24$ cubic unit cells, each unit cell containing one $\alpha$ (cube corner) and one $\beta$ (cube center) site. The initial bcc disordered configurations (A2 phase) were obtained by populating randomly the sites with the various species according to the overall composition. This high-temperature disordered solid solution was then cooled from $1600^{\circ} \mathrm{C}$, a value supposedly close to the experimental melting temperature of equimolar AlCrFeMnMo. The quenching process was simulated via the exchange Metropolis Monte-Carlo algorithm (MC), where atom pairs were allowed to exchange positions, these events being accepted or rejected according to the Boltzmann probability. To ensure that the obtained atomic configurations were representative of equilibrium, our calculations were run for $10^{6}$ jumps at each temperature step.

Order-disorder transitions correspond to rearrangements of the various chemical species on the $\alpha$ and $\beta$ sublattices. To characterize long-range order (LRO) in AlCrFeMnMo, which is of primary importance to follow the emergence of B2-type ordered phases from the A2 solid solution, the atomic distributions of the various chemical species were determined by LRO parameters $\eta_{\mathrm{i}}$, calculated from the following relation:

$\eta_{i}($ simulation $)=\frac{N_{i \alpha}-N_{i A v}}{N_{i A v}}$

$N_{i \alpha}$ is the number of atoms of type $i$ on the $\alpha$ sublattice, $N_{i A v}$ is the number of atoms of type $i$ on each sublattice, in the perfectly random solid solution:

$N_{i A \nu}=x_{i} N_{\alpha / \beta}$

$x_{i}$ is the molar fraction of element $i, N_{\alpha / \beta}$ is the number of sites in the $\alpha$ or $\beta$ sublattice. While the $\eta_{\text {i }}$ parameters can be unambiguously employed in single-phase systems, their interpretation becomes more tricky as soon as phase separation occurs, which in our case is systematically observed below some characteristic temperature (noted $\mathrm{T}_{\mathrm{ps}}$ below).

As short-range order (SRO) is also of great importance during phase transformations in HEAs, we additionally calculated the pair correlation functions $P_{i j}$ between various pairs of species. These quantities provide precious information about the HEA trends to form disordered (with no LRO) configurations in which chemical species $j$ occupies nearest-neighbor sites around atoms of species $i$. More precisely, the ratio $P_{i j} / x_{i} x_{j}$ between $P_{i j}$ and its uncorrelated expectation gives information about the trend for $\mathrm{i}$ and $\mathrm{j}$ elements to associate in first-neighbor pairs. It should be noted that $P_{i j}$ tends towards $x_{i} x_{j}$ in the high-temperature limit (no SRO remains in the perfectly random alloy). 
In the following section, the order-disorder and phase separation trends of AlCrFeMnMo are investigated by following the profiles of long-range order parameters $\left(\eta_{i}\right)$ and short-range order pair correlation functions $\left(P_{i j}\right)$ for decreasing temperatures. Since these profiles alone are not sufficient to inform unambiguously on these two major trends of HEAs, they are combined with the analysis of atomistic configurations. It should be noted that these "snapshots" become useful as soon as phase separation starts, in order to identify which chemical elements are present in each phase, since this information cannot be inferred from $\eta_{i}$ or $P_{i j}$ alone.

\section{Results}

\subsection{Preliminary: equimolar AlCrFeMnNi}

As mentioned above, the atomic-scale simulation approach adopted in this work has been shown in earlier studies to be efficient for the modeling of phase transformations in AlCoCrFeNi HEAs [29]. Moreover, AlCrFeMnNi, built by substituting Co by $\mathrm{Mn}$ in AlCoCrFeNi, provides a medium step between the latter system and our main target AlCrFeMnMo. This suggests beginning our study by applying the selected methodology to equimolar AlCrFeMnNi. In particular, this will provide a good insight into the influence of $\mathrm{Co} \rightarrow \mathrm{Mn}$ substitution in the alloy. We therefore describe first Monte-Carlo simulations on structural phase transformations in $\mathrm{AlCrFeMnNi}$, using the energy interaction parameters [23] listed in Table 1.

As already pointed out in earlier works [29], the identification of phases and ordering types in multicomponent concentrated alloys such HEAs is far from being straightforward. We have mentioned in the pre- vious section that long-range and short-range order parameters together may provide useful tools for such identification. However, as illustrated below, the reliability of these parameters is drastically reduced by the more or less gradual occurrence of phase separation with $\mathrm{T}$ lowering. As a consequence, the analysis of order parameters must be coupled with more direct inspection of snapshots of the system at various decreasing temperatures. Such a snapshot sequence for $\mathrm{AlCrFeMnNi}$ is presented on Fig. 1, while Figs. 2 and 3 display the related LRO and SRO profiles. Starting from the high-temperature A2-(Al,Cr, $\mathrm{Fe}, \mathrm{Mn}, \mathrm{Ni})$ single-phase system, the first thermal event is clearly visible on LRO around $T o \sim 900{ }^{\circ} \mathrm{C}$. More precisely, LRO indicates that two groups of elements are to be distinguished when cooling the solid solution: $\mathrm{Al}$ and $\mathrm{Ni}$ tend to adopt B2-type order, whereas $\mathrm{Cr}$, Fe and Mn keep their high-temperature random distribution. This corresponds to the emergence of a partially ordered $\mathrm{B} 2-(\mathrm{Al}, \mathrm{Cr}, \mathrm{Fe}, \mathrm{Mn})_{\alpha}(\mathrm{Cr}, \mathrm{Fe}, \mathrm{Mn}, \mathrm{Ni})_{\beta}$ single-phase system, as confirmed by direct inspection at $800 \mathrm{~K}$ (Fig. 1b) which shows no phase separation at this stage. Single-phase ordering is thus expected to occur prior to phase separation when cooling equimolar AlCrFeMnNi. Here and below, the various phases and orderings obtained at $\mathrm{T}$ lowering in the investigated alloys will be collected in Table 2. Interestingly, this Table evidences subtle alloy- and element-dependent ordering features, for instance when comparing $\mathrm{AlCoCrFeNi}$ and $\mathrm{AlCrFeMnNi}$ in their single-phase ordering domains (medium column in Table 2): the ordered distribution of $\mathrm{Cr}$ and $\mathrm{Fe}$ in $\mathrm{AlCoCrFeNi}$ becomes random distribution for these elements in $\mathrm{AlCrFeMnNi}$, a consequence of the replacement of Co with Mn.

\section{AlCrFeMnNi}

\section{a) $1600{ }^{\circ} \mathrm{C}$}

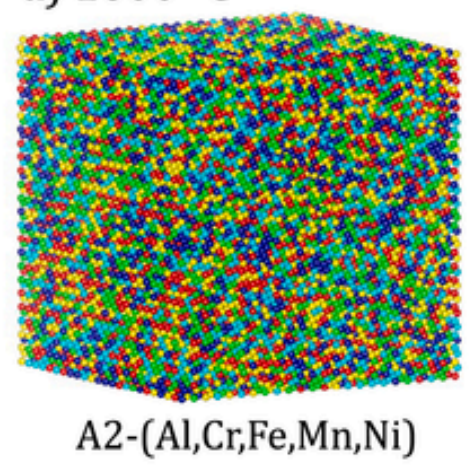

c) $600{ }^{\circ} \mathrm{C}$

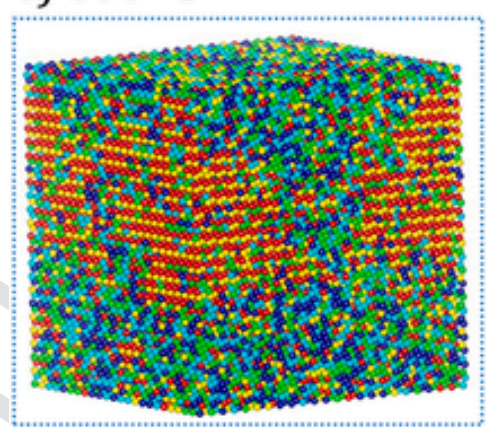

$$
\begin{gathered}
\mathrm{A} 2-(\mathrm{Cr}, \mathrm{Fe}, \mathrm{Mn})+\mathrm{B} 2-\mathrm{Al}_{\alpha} \mathrm{Ni}_{\beta}, \\
\text { "Dispersed two-phase state" }
\end{gathered}
$$

b) $800{ }^{\circ} \mathrm{C}$

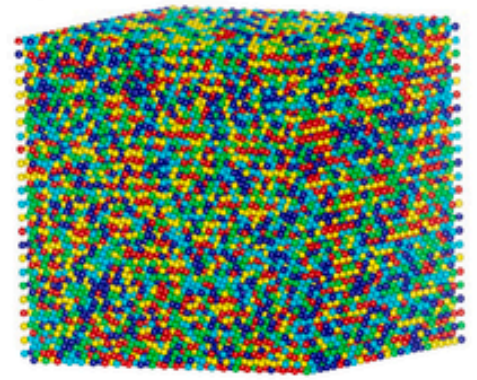

$\mathrm{B} 2-(\mathrm{Al}, \mathrm{Cr}, \mathrm{Fe}, \mathrm{Mn})_{\alpha}(\mathrm{Cr}, \mathrm{Fe}, \mathrm{Mn}, \mathrm{Ni})_{\beta}$

d) $27^{\circ} \mathrm{C}$

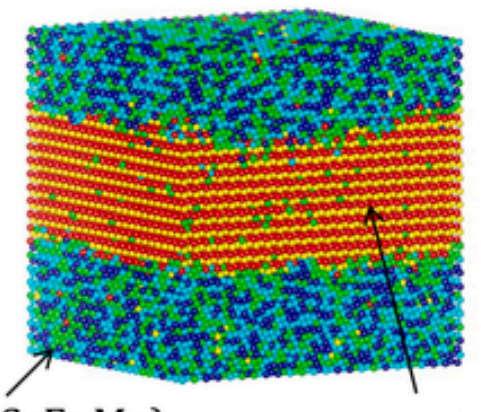

A2-(Cr,Fe,Mn)
$\mathrm{B} 2-\mathrm{Al}_{\alpha} \mathrm{Ni}_{\beta}$

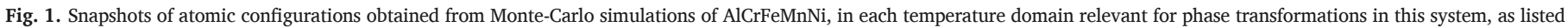
in Table 2. The color code for chemical species is shown on the chemical formula itself. 


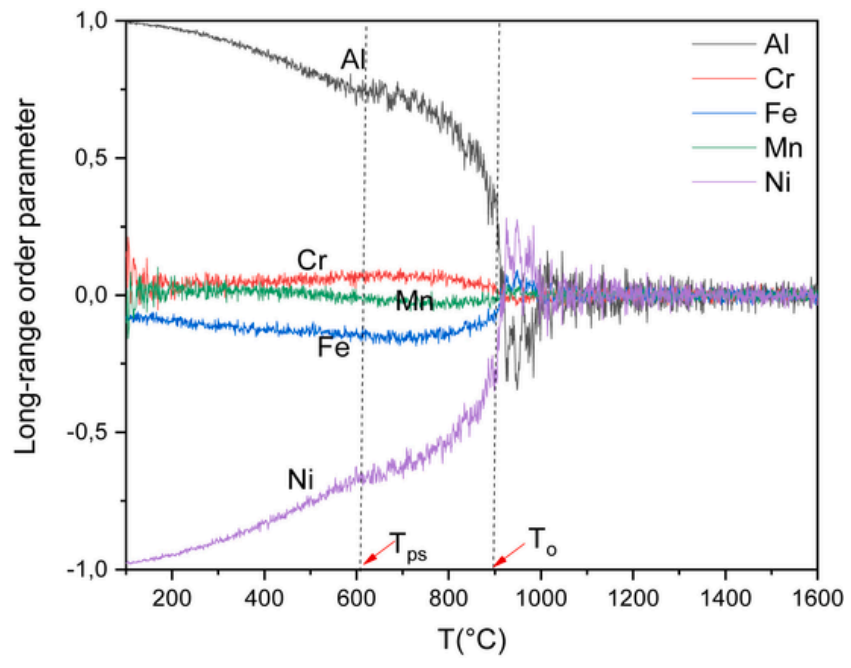

Fig. 2. Order-disorder analysis in equimolar AlCrFeMnNi: simulated long-range order parameters during cooling. $\mathrm{T}_{\mathrm{o}}$ corresponds to the order/disorder temperature, $\mathrm{T}_{\mathrm{ps}}$ is the phase separation onset temperature.

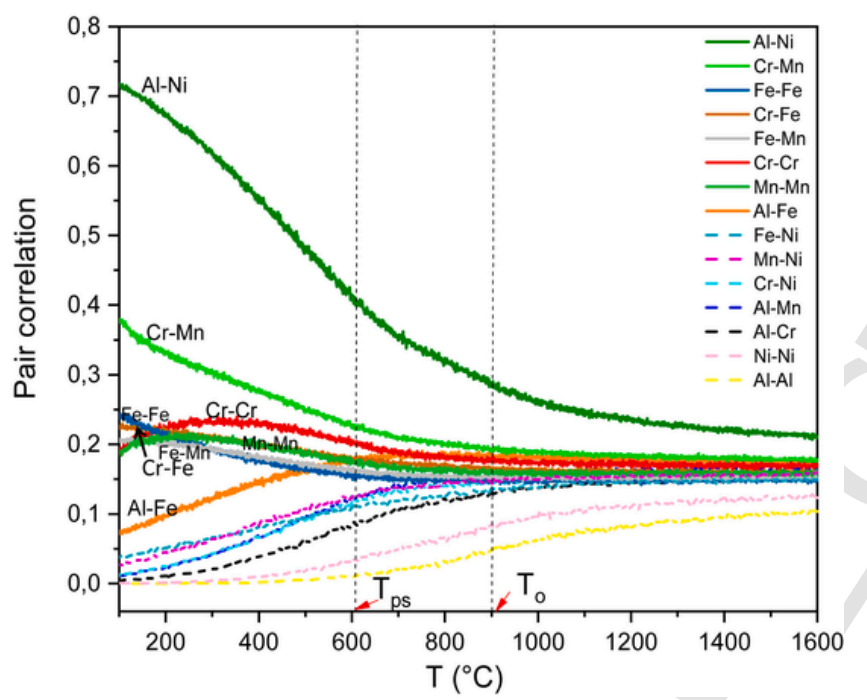

Fig. 3. Nearest-neighbor pair correlation functions in equimolar AlCrFeMnNi. $\mathrm{T}_{\mathrm{o}}$ corresponds to the order/disorder temperature. $\mathrm{T}_{\mathrm{ps}}$ is the phase separation onset temperature.

Further cooling makes the phase identification more tricky, as illustrated on Fig. 1c, which suggests that phase separation has started, however with subtle effects inducing the presence, in a more or less wide temperature range, of a "dispersed two-phase state" (DTPS). This occurrence of phase separation seems to reflect on LRO parameters: be- low some characteristic temperature noted $\mathrm{T}_{\mathrm{ps}}$ on the figures $\left(\mathrm{T}_{\mathrm{ps}} \sim 600^{\circ} \mathrm{C}\right.$ for $\mathrm{AlCrFeMnNi}$ ), these parameters show sudden changes of profiles with roughly inflection points at $\mathrm{T}_{\mathrm{ps}}$. It is worth mentioning here that increasing the simulation time (in isothermal mode at $600^{\circ} \mathrm{C}$, temperature of Fig. 1c) did not allow to get rid of the DTPS (no coalescence of the dispersed phase), which suggests that this is not a simulation artifact, but rather some possibly equilibrium state spuriously induced by specific features of the first-neighbor pair energy model used, for instance in relation with properties of the coherent interfaces between the two emerging phases. The temperature range of stability of the DTPS is difficult to estimate: in our simulations, it seems to be present below $\mathrm{T}_{\mathrm{ps}}$ down to room temperature.

At ambient temperature (Fig. 1d), the system is well phase-separated and adopts a two-layer structure. While Fig. 1d clearly allows to identify an ordered $\mathrm{B} 2-\mathrm{Al}_{\alpha} \mathrm{Ni}_{\beta}$ phase, the nature of the second phase, involving mainly $\mathrm{Cr}, \mathrm{Fe}$ and $\mathrm{Mn}$, is somewhat less straightforward, but is likely to correspond to disordered $\mathrm{A} 2-(\mathrm{Cr}, \mathrm{Fe}, \mathrm{Mn})$. In this unambiguously phase-separated state, further information on the phases formed can be gained from the SRO analysis. The pair correlation profiles (Fig. 3 ) thus reveal that $\mathrm{Al}$ has a strong trend to pair with $\mathrm{Ni}$, at values characteristic of the B2-(Al,Ni) phase (Fig. 1d). Fig. 3 also displays another group of correlation functions with lower values, i.e. for $\mathrm{Cr}-\mathrm{Cr}$, $\mathrm{Fe}-\mathrm{Fe}$, $\mathrm{Mn}-\mathrm{Mn}, \mathrm{Cr}-\mathrm{Mn}, \mathrm{Cr}-\mathrm{Fe}$ and Fe-Mn pairs, which nevertheless show significant increase at room temperature. These pairs should thus be related to the formation of a low-temperature A2-(Cr-Fe-Mn) phase, in reasonable agreement with the absence of any visible LRO within the (Cr-Fe-Mn) phase shown on Fig. 1d. The status of Al-Fe pairs is less clear, indicating that low amounts of these elements should be present in both phases. The remaining pairs roughly vanish at low temperature, since they connect elements belonging to distinct phases. This suggests a strong partitioning of $\mathrm{Cr}, \mathrm{Mn}$ and $\mathrm{Ni}$ between both phases. On the whole, this SRO analysis confirms that the system should be characterized as a mixture of $\mathrm{A} 2-(\mathrm{Cr}, \mathrm{Fe}, \mathrm{Mn})$ and $\mathrm{B} 2-\mathrm{Al}_{\alpha} \mathrm{Ni}_{\beta}$ phases in the low-temperature range (see Table 2). Noticeably, a disordered A2 phase is thus found to persist in $\mathrm{AlCrFeMnNi}$ at room temperature, a feature shared with previously studied AlCoCrFeNi (Table 2).

Our simulation results on equimolar $\mathrm{AlCrFeMnNi}$ are consistent with the experimental measurements of Zhang et al. [30] on $\mathrm{Al}_{\mathrm{x}} \mathrm{CrFeMnNi}$ $(x=0.5-0.8)$. In this experimental work, the XRD patterns showed the presence of an $\mathrm{A} 2$ phase enriched in $\mathrm{Cr}$ and $\mathrm{Fe}$, together with an ordered $\mathrm{B} 2-(\mathrm{Al}, \mathrm{Ni})$ rich phase. This agreement between experiments and simulations on $\mathrm{AlCrFeMnNi}$ confirms the reliability, already noted in earlier works on AlCoCrFeNi, of the model used here.

\subsection{AlCrFeMnMo system}

\subsubsection{Equimolar alloy}

Following the same scheme for analysis, we now consider the case of equimolar AlCrFeMnMo. The snapshots at relevant temperatures

Table 2

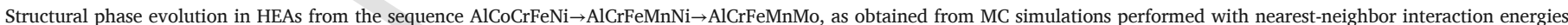
extracted from ab initio calculations [23].

\begin{tabular}{|c|c|c|c|}
\hline HEA & Predicted phases & & \\
\hline \multirow[t]{2}{*}{ AlCoCrFeNi29 } & $\rightarrow$ TEMPERATURE LOWERING & & \\
\hline & $\mathrm{A} 2-(\mathrm{Al}, \mathrm{Co}, \mathrm{Cr}, \mathrm{Fe}, \mathrm{Ni})$ & $\mathrm{B} 2-(\mathrm{Al}, \mathrm{Cr}, \mathrm{Fe})_{\alpha}(\mathrm{Co}, \mathrm{Ni})_{\beta}$ & $\mathrm{A} 2-(\mathrm{Cr}, \mathrm{Fe})+\mathrm{B} 2-\mathrm{Al}_{\alpha}(\mathrm{Co}, \mathrm{Ni})_{\beta}$ \\
\hline \multirow{2}{*}{$\mathrm{AlCrFeMnNi}$} & Replacing Co with Mn & & \\
\hline & $\mathrm{A} 2-(\mathrm{Al}, \mathrm{Cr}, \mathrm{Fe}, \mathrm{Mn}, \mathrm{Ni})$ & $\mathrm{B} 2-(\mathrm{Al}, \mathrm{Cr}, \mathrm{Fe}, \mathrm{Mn})_{\alpha}(\mathrm{Cr}, \mathrm{Fe}, \mathrm{Mn}, \mathrm{Ni})_{\beta}$ & \\
\hline \multirow[t]{2}{*}{ AlCrFeMnMo } & Replacing Ni with Mo & & \\
\hline & $\mathrm{A} 2-(\mathrm{Al}, \mathrm{Cr}, \mathrm{Fe}, \mathrm{Mn}, \mathrm{Mo})$ & $\mathrm{B} 2-(\mathrm{Cr}, \mathrm{Fe}, \mathrm{Mn})_{\alpha}(\mathrm{Al}, \mathrm{Cr}, \mathrm{Mo})_{\beta}$ & $\mathrm{B} 2-(\mathrm{Cr}, \mathrm{Mn})_{\alpha 1}(\mathrm{Al}, \mathrm{Cr})_{\beta 1}+\mathrm{B} 2-\mathrm{Fe}_{\alpha 2} \mathrm{Mo}_{\beta 2}$ \\
\hline \multirow[t]{2}{*}{$\mathrm{Al}_{22} \mathrm{Cr}_{22} \mathrm{Fe}_{29} \mathrm{Mn}_{20} \mathrm{Mo}_{7}$} & Partially replacing Mo with Fe & & \\
\hline & $\mathrm{A} 2-(\mathrm{Al}, \mathrm{Cr}, \mathrm{Fe}, \mathrm{Mn}, \mathrm{Mo})$ & $\mathrm{B} 2-(\mathrm{Fe}, \mathrm{Mn})_{\alpha}(\mathrm{Al}, \mathrm{Cr}, \mathrm{Mo})_{\beta}$ & $\mathrm{B} 2-\mathrm{Cr}_{\alpha 1} \mathrm{Mn}_{\beta 1}+\mathrm{B} 2-\mathrm{Fe}_{\alpha 2}(\mathrm{Al}, \mathrm{Mo})_{\beta 2}$ \\
\hline
\end{tabular}


$\left(1600^{\circ} \mathrm{C}, 800^{\circ} \mathrm{C}, 600{ }^{\circ} \mathrm{C}, 27^{\circ} \mathrm{C}\right)$ for this alloy, together with the LRO and SRO profiles, are displayed on Fig. 4, 5 and 6 respectively. As for Al$\mathrm{CrFeMnNi}$, the first event detected when cooling AlCrFeMnMo from the high-temperature A2-(Al,Cr,Fe,Mn,Mo) solid solution (Fig. 4a) is single-phase long-range ordering at $\mathrm{T}_{\mathrm{O}} \sim 800^{\circ} \mathrm{C}$, as can be checked from Figs. $4 \mathrm{~b}$ and $5 . \mathrm{Cr}$ is however found to retain random distribution, its LRO parameter remaining negligible. Conversely, the other elements tend to order, since $\mathrm{Fe}$ and $\mathrm{Mn}$ atoms (resp. $\mathrm{Al}$ and Mo) occupy the $\alpha$ (resp. $\beta$ ) sublattice. Moreover, as temperature decreases, long-range ordering is more significant for $\mathrm{Fe}$ and Mo than for $\mathrm{Al}$ and $\mathrm{Mn}$. On the whole, Fig. 5 allows to infer the formation, prior to any phase separation, of a partially ordered single-phase $\mathrm{B} 2-(\mathrm{Cr}, \mathrm{Fe}, \mathrm{Mn})_{\alpha}(\mathrm{Al}, \mathrm{Cr}, \mathrm{Mo})_{\beta}$ at To $\sim 800^{\circ} \mathrm{C}$.

As in the previous section, further cooling leads to an intermediate temperature domain (inflection points on $\mathrm{LRO}$ at $\mathrm{T}_{\mathrm{ps}} \sim 600^{\circ} \mathrm{C}$ ), in which equimolar AlCrFeMnMo adopts a seemingly DTPS (Fig. 4c), though to an extent somewhat lower than AlCrFeMnNi (compare with Fig. 1c). As before, we thus skip this domain for analysis and consider directly ambient-T properties (Fig. 4d). In this temperature range, the system is found to decompose into a well-defined two-phase mixture: Fig. $4 \mathrm{~d}$ unambiguously allows to identify the phases as $\mathrm{B} 2-(\mathrm{Cr}, \mathrm{Mn})_{\alpha 1}(\mathrm{Al}, \mathrm{Cr})_{\beta 1}$ and $\mathrm{B} 2-\mathrm{Fe}_{\alpha 2} \mathrm{Mo}_{\beta 2}$. The indices $\alpha 1, \alpha 2, \beta 1, \beta 2$ refer to the sublattices $\alpha$ and $\beta$ of phases 1 and 2 .

Here again, complementary information can be gained from inspection of SRO properties (Fig. 6). Fe-Mo pairs increase sharply, which corresponds to the formation of the low-T B2-(Fe,Mo) phase (Fig. 4). Moreover, Al preferentially pairs with $\mathrm{Mn}$, whereas $\mathrm{Al}-\mathrm{Cr}, \mathrm{Cr}-\mathrm{Mn}$ and $\mathrm{Cr}-\mathrm{Cr}$ pairs increase slightly, in agreement with the formation of the low-T B2-(Al,Cr,Mn) phase with $\mathrm{Cr}$ in random distribution (Fig. 4). Other pair functions vanish during cooling, for pairs of elements either belonging to distinct phases, or sharing the same sublattice in a given low-T B2 phase.
The currently low amount of knowledge on AlCrFeMnMo HEAs makes it especially interesting to compare our atomic-scale simulation results with previous CALPHAD calculations performed by Senkov [27]. In particular, whereas the latter work suggested the stability of the A2 solid solution and a non-cubic $\mathrm{AlMo}_{3}$ ordered compound (which cannot be predicted from our rigid-lattice model), our MC calculations rather show the presence (below $600{ }^{\circ} \mathrm{C}$ ) of a two-phase cubic mixture of $\mathrm{B} 2-(\mathrm{Cr}, \mathrm{Mn})_{\alpha 1}(\mathrm{Al}, \mathrm{Cr})_{\beta 1}$ and $\mathrm{B} 2-\mathrm{Fe}_{\alpha 2} \mathrm{Mo}_{\beta 2}$ (see Table 2). Further investigations would be needed here, in order to determine the respective roles of the atomic-scale energy model and phenomenological thermodynamic database in these discrepancies. In particular, atomic-scale modeling of $\mathrm{AlMo}_{3}$ combined with the available first-neighbor pair model for bcc-based structures may improve our insight into these issues. On the other hand, recent experimental measurements [26] on AlCrFeMnMo showed the presence of two bcc-based A2 phases (a Cr-rich one and a Mo-rich one), also at odds with our simulation findings. Apart from deficiencies in the first-neighbor pair model used above (due to the successful behavior of the model for AlCoCrFeNi and AlCrFeMnNi, such deficiencies might be related to Mo), a relevant attempt to explain such discrepancies between our theoretical predictions and earlier experimental findings on $\mathrm{AlCrFeMnMo}$ may be related to the uncertainty on the composition of alloys elaborated in practice. Such a hypothesis would be consistent with the possibility that mixtures A2' $+\mathrm{A} 2$ '" of disordered phases be stable in particular domains of composition, unexpectedly produced in practice due to unavoidable fluctuations in the elaboration processes. To answer such questions for AlCrFeMnMo, a full Monte-Carlo exploration of the composition space for this bcc-based quinary system would be highly fruitful in principle, but this remains an unachievable task. Nevertheless, in order to get a first insight into this issue, we now focus on a specific Fe-enriched and Mo-depleted "optimized" alloy previously selected on empirical grounds.

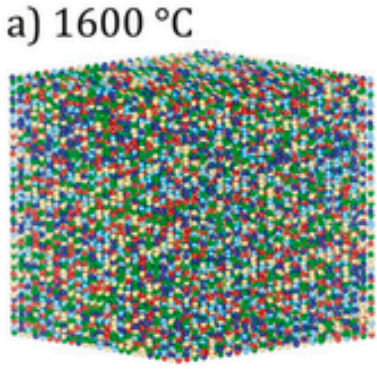

A2-AlCrFeMnMo

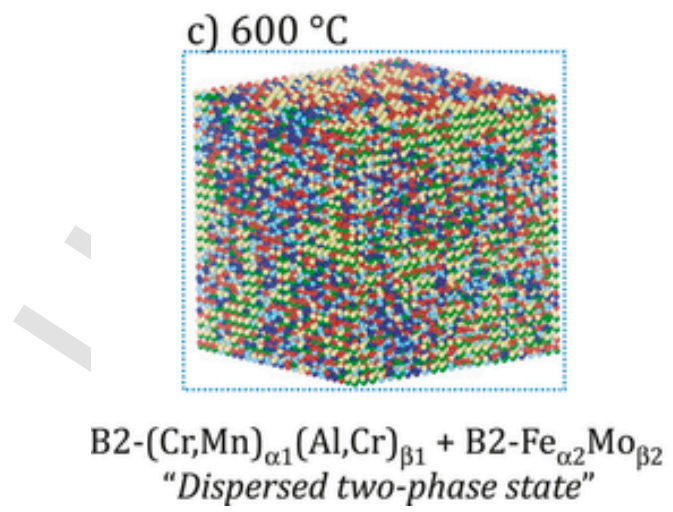

b) $800^{\circ} \mathrm{C}$

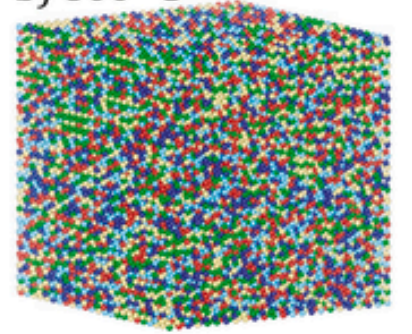

$\mathrm{B} 2-(\mathrm{Cr}, \mathrm{Fe}, \mathrm{Mn})_{\alpha}(\mathrm{Al}, \mathrm{Cr}, \mathrm{Mo})_{\beta}$
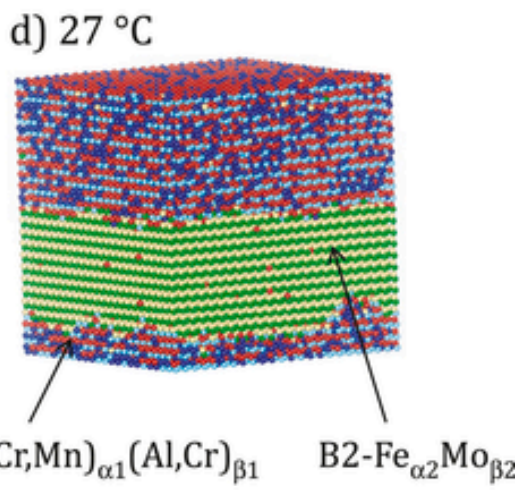

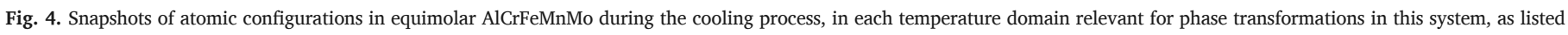
in Table 2. 


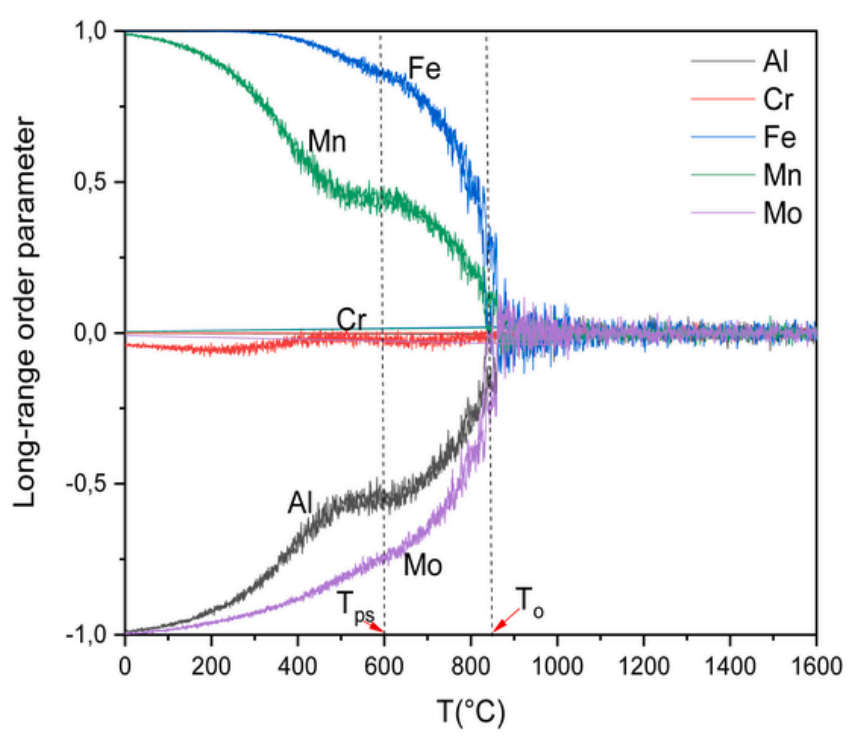

Fig. 5. Variation of element-specific long-range order parameters with temperature in equimolar AlCrFeMnMo. $\mathrm{T}_{\mathrm{o}}$ corresponds to the order/disorder temperature, $\mathrm{T}_{\mathrm{ps}}$ is the phase separation onset temperature.

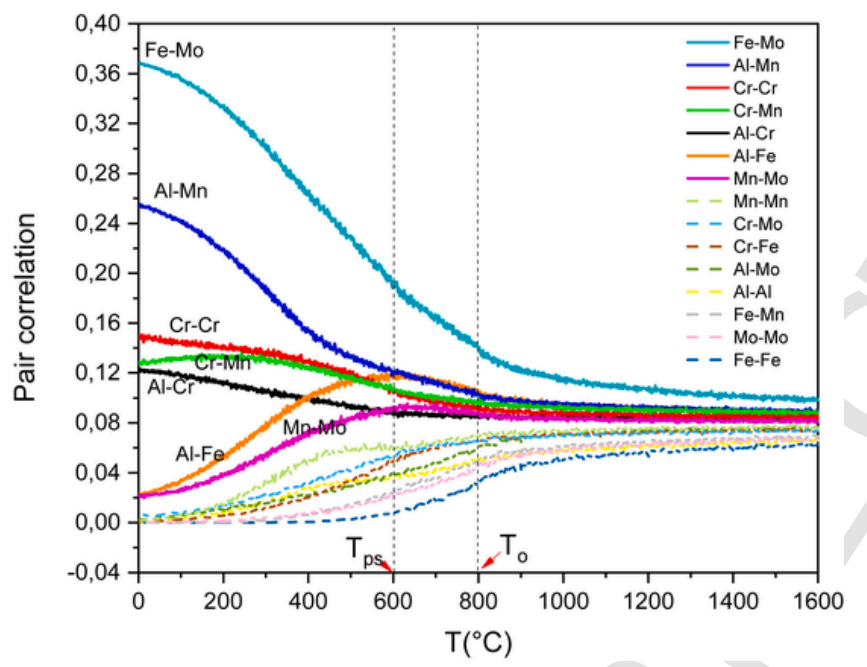

Fig. 6. Pair correlation function variation with temperature in the equimolar $\mathrm{AlCrFeMnMo}$ HEA, $\mathrm{T}_{\mathrm{ps}}$ is the phase separation onset temperature.

\subsubsection{Optimized alloy $\mathrm{Al}_{22} \mathrm{Cr}_{22} \mathrm{Fe}_{29} \mathrm{Mn}_{20} \mathrm{Mo}_{7}$}

Following the reference case of equimolar AlCrFeMnMo, we then consider an "optimized" $\mathrm{Al}_{22} \mathrm{Cr}_{22} \mathrm{Fe}_{29} \mathrm{Mn}_{20} \mathrm{Mo}_{7}$ alloy [26]. The issue investigated here concerns the effect, on phase formation and ordering, of a selected composition change around the equimolar reference alloy, as usually suggested in practice by more or less heuristic optimization schemes, such as the parametric approach, or through more elaborate thermodynamic calculations from phenomenological databases.

Comparing Figs. $7 \mathrm{~b}$ and 8, our MC simulations predict that, at this composition, the high-temperature $\mathrm{A} 2-(\mathrm{Al}, \mathrm{Cr}, \mathrm{Fe}, \mathrm{Mn}, \mathrm{Mo})$ solid solution firstly transforms to a partially ordered $\mathrm{B} 2-(\mathrm{Fe}, \mathrm{Mn})_{\alpha}(\mathrm{Al}, \mathrm{Cr}, \mathrm{Mo})_{\beta}$ single phase. From Fig. 8, the order-disorder transition temperature, To $\sim 750^{\circ} \mathrm{C}$, is slightly lower than that found for the equimolar alloy ( To $\sim 800^{\circ} \mathrm{C}$ ). Moreover, $\mathrm{Cr}$ atoms tend to an ordered state, while $\mathrm{Cr}$ was found to remain in random distribution in the equimolar alloy. On the contrary Al, Fe, Mn and Mo have similar ordered behaviors at both compositions investigated.
Further cooling (Fig. 7c) entails the emergence of a DTPS though at temperature $\mathrm{T}_{\mathrm{ps}} \sim 400{ }^{\circ} \mathrm{C}$ (inflection points on Fig. 8) significantly lower than in the equimolar alloy. At ambient temperature, phase separation is clearly observed with the formation of a two-layer system consisting of phases which can be labeled as $\mathrm{B} 2-\mathrm{Cr}_{\alpha 1} \mathrm{Mn}_{\beta 1}$ and $\mathrm{B} 2-\mathrm{Fe}_{\alpha 2}(\mathrm{Al}, \mathrm{Mo})_{\beta 2}$. While these ordering patterns and chemical compositions may roughly be inferred from direct inspection (Fig. 7d), they are confirmed by examining pair correlations. Fig. 9, together with Fig. 6, shows that dominant pairs differ significantly in the equimolar and optimized alloys, since at ambient temperature, Fe-Mo and Al-Mn are no longer dominant pairs in the latter one. Al-Fe pairs increase sharply, which reflects the formation of a B2-type ordered phase with $\mathrm{Fe}$ and $\mathrm{Al}$ on each sublattice. Noticeably, the signature of this highly documented $\mathrm{B} 2-\mathrm{Fe}_{\alpha} \mathrm{Al}_{\beta}$ phase is much clearer in the optimized alloy, since it is found to be stable even at low $\mathrm{T}$, instead of its more transient character in the equimolar case (Table 2). In the optimized alloy, Fe-Mo pairs are also present, though at a level reduced with respect to equimolar composition, confirming the presence of $\mathrm{B} 2-(\mathrm{Al}, \mathrm{Fe}, \mathrm{Mo})$. $\mathrm{Cr}-\mathrm{Mn}$ pairs also increase, which corresponds to the $\mathrm{B} 2-(\mathrm{Cr}, \mathrm{Mn})$ phase clearly visible at ambient temperature (Fig. 7d). It is a remarkable feature that a rather moderate composition change may induce drastic changes of behavior, in particular for $\mathrm{Cr}$, which switches from random distribution to strong order with Mn (Table 2). Finally, Cr-Cr, Al-Mn and Cr-Fe form a group of secondary pairs, the exact status of which is however difficult to assess, while remaining pairs strictly vanish at low temperature.

On the whole, our simulations indicate that increasing the iron content and decreasing the molybdenum content in AlCrFeMnMo HEAs favors ordering for chromium and phase change for aluminum, namely $\mathrm{B} 2-(\mathrm{Al}, \mathrm{Fe}, \mathrm{Mo})$ instead of $\mathrm{B} 2-(\mathrm{Al}, \mathrm{Cr}, \mathrm{Mn})$. Noticeably, this composition change also stabilizes the well-known $\mathrm{B} 2-\mathrm{FeAl}$ phase at low temperature. Moreover, it is important to underline that the two-phase mixtures obtained at low temperatures in AlCrFeMnMo for the couple of compositions studied include at least one ordered B2-type phase, contrary to previous experimental results [26]. This discrepancy, which may seem surprising since the energy model used here was found reliable for AlCoCrFeNi [29] and AlCrFeMnNi [30], may be due to deficiencies in the Mo-X interactions from Table 1. Alternatively, this might also be explained by the presence of other elements possibly acting as potential sources of disorder. Indeed, during mechanical alloying of AlCrFeMnMo, carbon contamination was noticed [26], which can significantly affect phase stability properties in this HEA family.

\section{Discussion}

In multicomponent alloys as those studied in this work, entropy effects are of main importance when phase stability is considered. For this reason, in this part, we first discuss the role of configurational entropy in order/disorder transitions and phase decompositions in the above HEAs, on the basis of the LRO and SRO profiles obtained previously. In presence of LRO, the configurational entropy can be expressed in terms of parameters $\eta_{i}$ as follows [28]:

$$
\begin{aligned}
S_{L R O}=-\frac{R}{2} \sum_{i=1}^{n}\left\{x_{i}(1+\right. & \left.\eta_{i}\right) \ln \left[x_{i}\left(1+\eta_{i}\right)\right] \\
& \left.+x_{i}\left(1-\eta_{i}\right) \ln \left[x_{i}\left(1-\eta_{i}\right)\right]\right\}
\end{aligned}
$$

where the summation runs over the $n=5$ chemical elements, $R$ is the gas constant, and $x_{i}$ is the molar fraction of element $i$.

In presence of SRO but no LRO, the entropy can be quantified via the notion of "mutual information" embodied in the $\mathrm{P}_{\mathrm{ij}}$ parameters. Therefore, in this picture, the configurational entropy for ideal mixing ( $S_{\text {mix }}^{\text {conf }}=-R \sum_{i=1}^{n} x_{i} \ln \left(x_{i}\right)$ ) is reduced by this "mutual information" content 


\section{$\mathrm{Al}_{22} \mathrm{Cr}_{22} \mathrm{Fe}_{29} \mathrm{Mn}_{20} \mathrm{Vlo}_{7}$}

a) $1600^{\circ} \mathrm{C}$

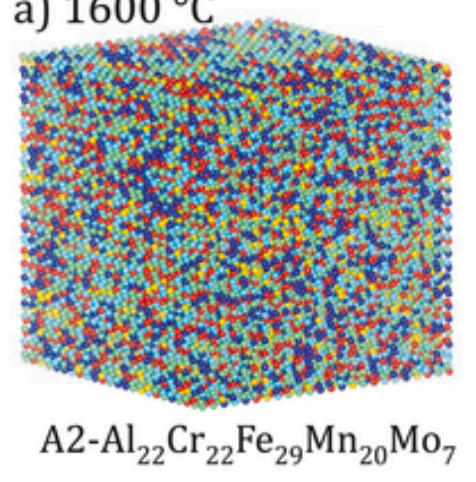

c) $400{ }^{\circ} \mathrm{C}$

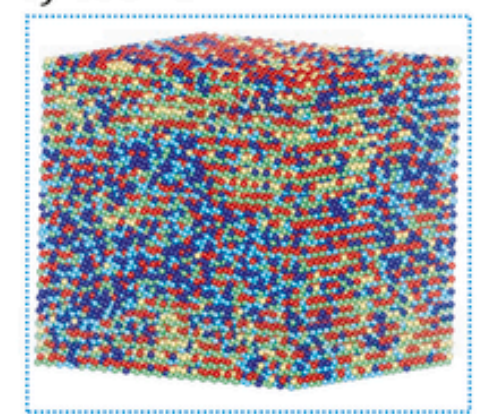

\section{$\mathrm{B} 2-\mathrm{Cr}_{\alpha 1} \mathrm{Mn}_{\beta 1}+\mathrm{B} 2-\mathrm{Fe}_{\alpha 2}(\mathrm{Al}, \mathrm{Mo})_{\beta 2}$ \\ "Dispersed two-phase state"}

b) $700^{\circ} \mathrm{C}$

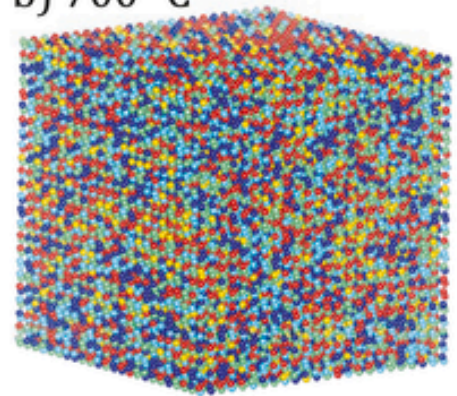

$\mathrm{B} 2-(\mathrm{Fe}, \mathrm{Mn})_{\alpha}(\mathrm{Al}, \mathrm{Cr}, \mathrm{Mo})_{\beta}$

d) $27^{\circ} \mathrm{C}$

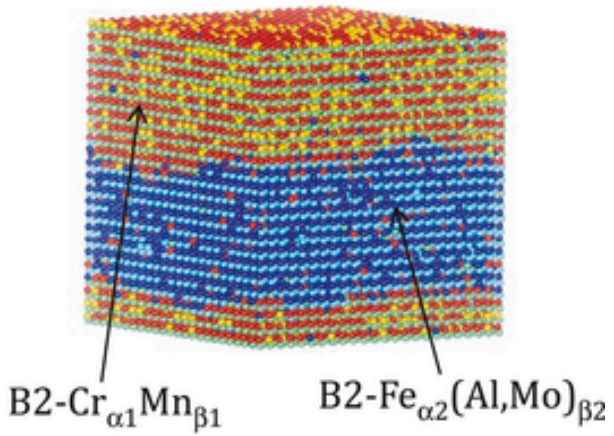

Fig. 7. Snapshots of atomic configurations in $\mathrm{Al}_{22} \mathrm{Cr}_{22} \mathrm{Fe}_{29} \mathrm{Mn}_{20} \mathrm{Mo}_{7}$ in each temperature domain relevant for phase transformations in this system, as listed in Table 2 .

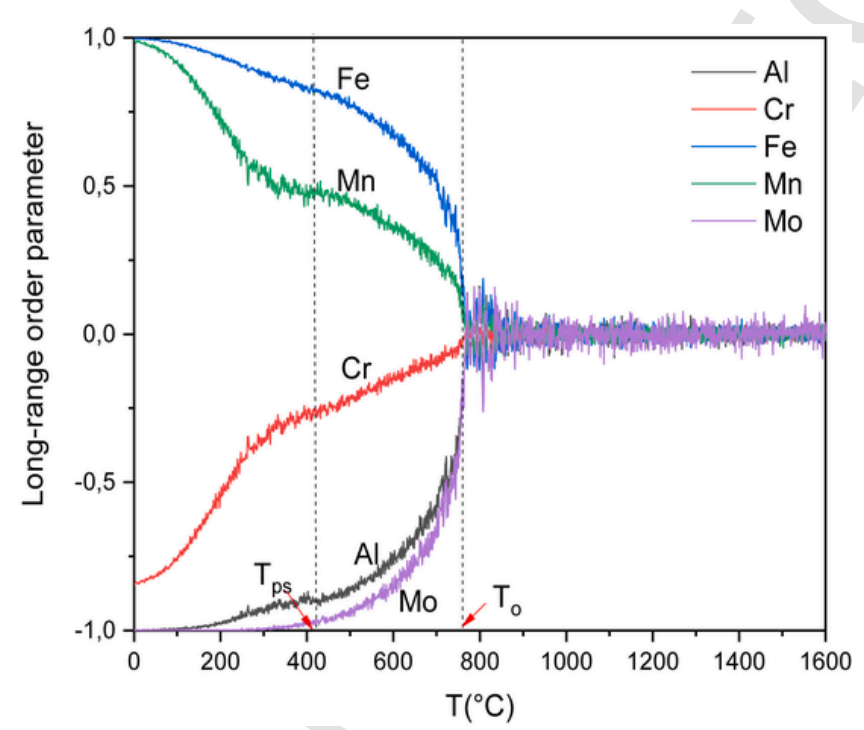

Fig. 8. Monte-Carlo simulations of long-range order parameters in $\mathrm{Al}_{22} \mathrm{Cr}_{22} \mathrm{Fe}_{29} \mathrm{Mn}_{20} \mathrm{Mo}_{7}$. $\mathrm{T}_{\mathrm{o}}$ corresponds to the order/disorder temperature, $\mathrm{T}_{\mathrm{ps}}$ is the phase separation onset temperature.

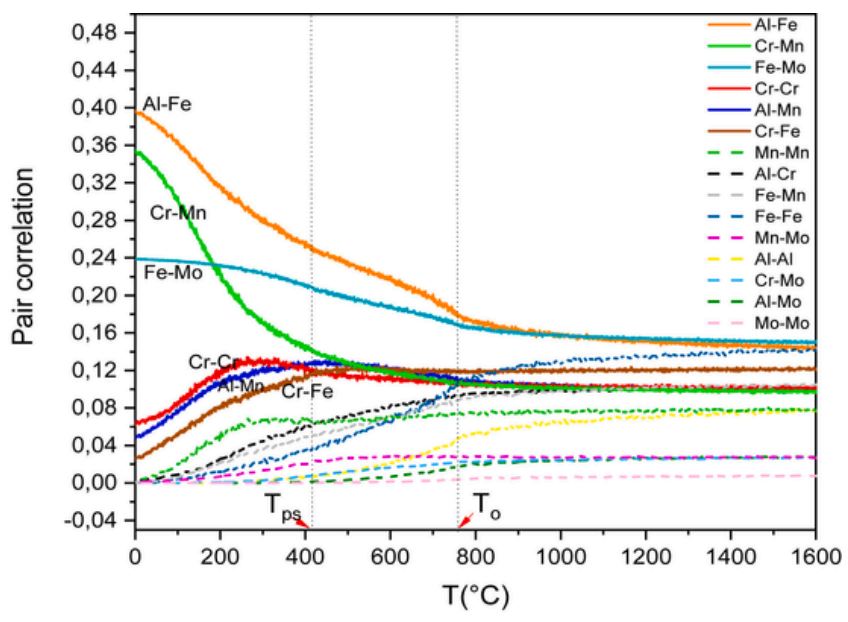

Fig. 9. Short-range order analysis: pair correlation function variation during quenching in $\mathrm{Al}_{22} \mathrm{Cr}_{22} \mathrm{Fe}_{29} \mathrm{Mn}_{20} \mathrm{Mo}_{7} \mathrm{~T}_{\mathrm{ps}}$ is the phase separation onset temperature.

to yield the SRO entropy [31,32]:

$S_{S R O}=-R \sum_{i=1}^{n} x_{i} \ln \left(x_{i}\right)-4 R \sum_{i j} P_{i j} \ln \left(\frac{P_{i j}}{x_{i} x_{j}}\right)$

Fig. 10 displays the LRO and SRO entropies thus obtained for the AlCrFeMnNi, AlCrFeMnMo and $\mathrm{Al}_{22} \mathrm{Cr}_{22} \mathrm{Fe}_{29} \mathrm{Mn}_{20} \mathrm{Mo}_{7}$ alloys. As a first remark, these entropies yield similar $T_{o}$ values as those obtained above from order parameters. Noticeably, since this agreement is good be- 

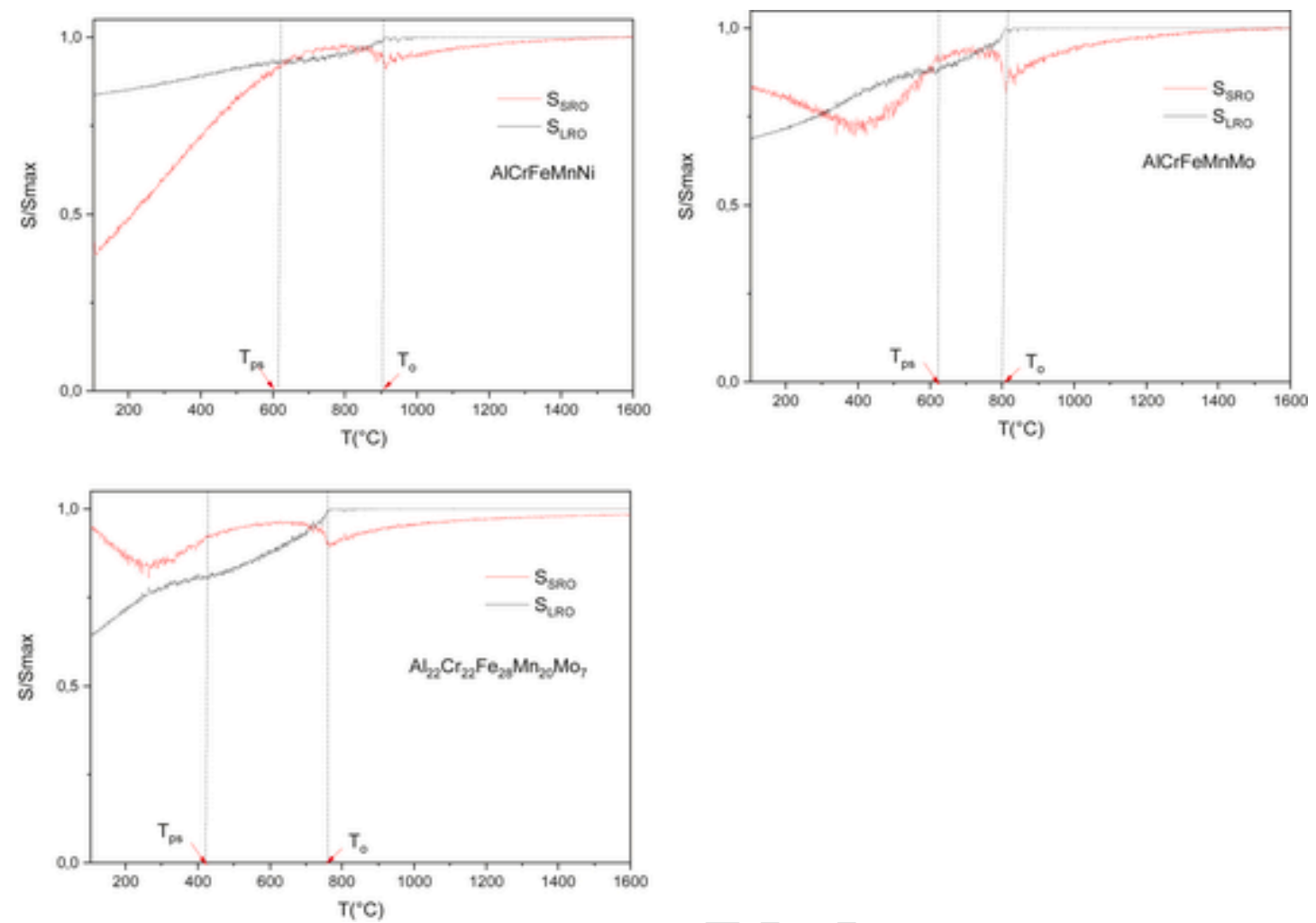

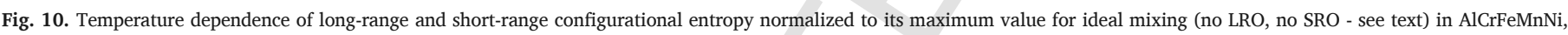
AlCrFeMnMo and $\mathrm{Al}_{22} \mathrm{Cr}_{22} \mathrm{Fe}_{29} \mathrm{Mn}_{20} \mathrm{Mo}_{7}$. $\mathrm{T}_{\mathrm{o}}$ corresponds to the order/disorder temperature, $\mathrm{T}_{\mathrm{ps}}$ is the phase separation onset temperature.

tween LRO and SRO profiles, $\mathrm{S}_{\mathrm{SRO}}$ can thus be regarded as a reliable indicator, more sensitive than separate $\mathrm{P}_{\mathrm{ij}}$, for LRO onset. Below $T_{o}$, the LRO entropy profiles consistently show monotonous decrease for all alloys. Conversely, the interpretation of SRO entropies is more difficult, probably due to the progressive character of phase separation (Figs. $1 \mathrm{c}, 4 \mathrm{c}$ and $7 \mathrm{c}$ ) and the related alloy-dependent partitioning of elements between both phases. As a consequence, the $\mathrm{S}_{\mathrm{SRO}}$ evolution for $\mathrm{AlCr}$ FeMnNi significantly differs from those obtained for both AlCrFeMnMo alloys, which are found similar to each other. Thermodynamic integration approaches, in principle more exact than expressions (3) and (4) could help in interpreting these intricate SRO entropy profiles, but the handling of such exact schemes should probably be made exceedingly difficult by the simultaneous presence of two phases. As regards the links between these analytic entropies and the second characteristic HEA temperature $\mathrm{T}_{\mathrm{ps}}$ (the values of which reported on Fig. 10 are those previously determined in section III), these links remain difficult to establish, and LRO as well as SRO entropies cannot be regarded as efficient indicators of phase separation. It is nevertheless interesting to point out an inflection for SRO entropy near $\mathrm{T}_{\mathrm{ps}}$ for both AlCrFeMnMo alloys, but no such inflection exists for AlCrFeMnNi.

Pursuing our analysis of phase stability, it is interesting to come back on the ab initio interatomic energy parameters (Table 1 , also illustrated on Fig. 11) and their relation with the obtained phases at various temperatures. Noticeably, the addition of $\mathrm{Al}$ favors interaction with all elements with a particularly strong interaction with Ni (Al-
$\mathrm{Ni}=-8.15 \mathrm{~kJ} / \mathrm{mol}$ ). This suggests the formation of an ordered B2-type $\mathrm{Al}-\mathrm{Ni}$ phase in $\mathrm{AlCrFeMnNi}$ at low temperatures, as found with our MC simulations and with experimental measurements [30]. Likewise, since the Fe-Mo interaction parameter is high $(-5.82 \mathrm{~kJ} / \mathrm{mol})$, this suggests the formation of an ordered Fe-Mo phase at low temperatures, which is also obtained with our MC simulations in AlCrFeMnMo alloys. A similar analysis can be performed on the most important next two parameters (Al-Fe and Al-Mn), leading to the formation of ordered phases, as can be checked from Table 2. Notice however that the Al-Fe ordered phase is strongly composition-sensitive (it appears only in the optimized alloy), which emphasizes the limits of this qualitative analysis based solely on interaction parameters.

Besides the numerical values of pair interactions, the validity of our results can also be questioned due to the use of a simple energy model relying only on short-range pairs. This choice, which has been widely discussed in the literature [33], nevertheless appears reasonable given the enormous complexity of HEAs. Remarkably, this simple modeling proved its ability to evidence striking trends, e.g. concerning possible deficiencies in Mo-X interactions.

As a final issue, it should be pointed out that, since Monte-Carlo simulations, thermodynamically exact, allow to explore only small portions of the composition space, it would be interesting to jointly develop mean-field analytical approaches, in order to gain an overview of the behavior of HEAs at all compositions.

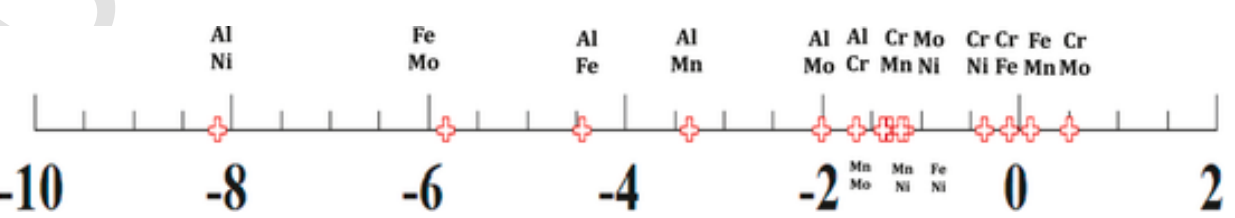

Fig. 11. Nearest-neighbor interaction energies $(\mathrm{kJ} / \mathrm{mol})$ reported in Table 1 (data obtained from earlier first-principles calculations [23]). 


\section{Conclusion}

The present work was devoted to atomic-scale investigations of ordering and phase separation in a new family of HEAs based on the AlCrFeMnMo system, using nearest-neighbor energy parameters extracted from ab initio calculations and Monte-Carlo simulations. Applying the approach to $\mathrm{AlCrFeMnNi}$, the model predicts the presence of A2/B2 phases, in agreement with previously published experimental studies. Conversely, in AlCrFeMnMo, two-phase mixtures involving only B2-type order are obtained at ambient temperature, at odds with the few available results. Comparing the various HEAs investigated suggests that the interaction energy parameters involving molybdenum might be inadequate. Further experimental measurements and mean-field analytical approaches should be developed in order to gain an overview of the behavior of HEAs in wider composition domains.

\section{Declaration of Competing Interest}

The authors declare that they have no known competing financial interests or personal relationships that could have appeared to influence the work reported in this paper.

\section{Acknowledgments}

The authors would like to thank the Interreg France-Wallonie-Vlaanderen project "Allihentrop" for financial support. The Chevreul Institute is thanked for its help in the development of this work through the ARCHI-CM project supported by the "Ministère de l'Enseignement Supérieur de la Recherche et de l'Innovation", the region "Hauts-de-France", the ERDF program of the European Union and the "Métropole Européenne de Lille".

\section{References}

[1] B.E. MacDonald, Z. Fu, B. Zheng, W. Chen, Y. Lin, F. Chen, L. Zhang, J. Ivanisenko, Y. Zhou, H. Hahn, E.J. Lavernia, Recent progress in high entropy alloy research, JOM 69 (10) (2017) 2024-2031.

[2] J.W. Yeh, Recent progress in high-entropy alloys, Ann. De. Chim.-Sci. Des. Matér. 31 (6) (2006) 633-648.

[3] W.H. Wu, C.C. Yang, J.W. Yeh, Industrial development of high-entropy alloys, Ann. De. Chim.-Sci. Des. Matér. 31 (6) (2006) 737-747.

[4] Z. Li, K.G. Pradeep, Y. Deng, D. Raabe, C.C. Tasan, Metastable high-entropy dual-phase alloys overcome the strength-ductility trade-off, Nature 534 (2016) $227-230$.

[5] J.H. Zhao, X.L. Ji, Y.P. Shan, Y. Fu, Z. Yao, On the microstructure and erosion-corrosion resistance of AlCrFeCoNiCu high-entropy alloy via annealing treatment, Mater. Sci. Technol. 32 (2016) 1271-1275.

[6] M. Yang, X.J. Liu, H.H. Ruan, Y. Wu, H. Wang, Z.P. Lu, High thermal stability and sluggish crystallization kinetics of high-entropy bulk metallic glasses, J. Appl. Phys. 119 (2016) 245112.

[7] W.-Y. Ching, S. San, J. Brechtl, R. Sakidja, M. Zhang, P.K. Liaw, Fundamental electronic structure and multiatomic bonding in 13 biocompatible high-entropy alloys, NPJ Comput. Mater. 6 (2020) 1-10.

[8] Y. Chou, J. Yeh, H. Shih, The effect of molybdenum on the corrosion behaviour of the high-entropy alloys Co1:5CrFeNi1:5Ti0:5Mox in aqueous environments, Corros. Sci. 52 (2010) 2571-2581.

[9] C. Chen, H. Zhang, Y. Fan, W. Zhang, R. Wei, T. Wang, T. Zhang, F. Li, A nove ultrafine-grained high entropy alloy with excellent combination of mechanical and soft magnetic properties, J. Magn. Magn. Mater. 502 (2020) 166513.

[10] C. Niu, A.J. Zaddach, C.C. Koch, D.L. Irving, , First principles exploration of near equiatomic NiFeCrCo high entropy alloys, J. Alloy. Compd. 672 (2016) 510-520.

[11] M.S. Titus, R.K. Rhein, P.B. Wells, P.C. Dodge, G.B. Viswanathan, M.J. Mills, A. Van der Ven, T.M. Pollock, Solute segregation and deviation from bulk thermodynamics at nanoscale crystalline defects, Sci. Adv. 2 (12) (2016) 1601796.

[12] E.J. Pickering, N.G. Jones, High-entropy alloys: a critical assessment of their founding principles and future prospects, Int. Mater. Rev. 61 (3) (2016) 183-202.

[13] Y. Zhang, G.M. Stocks, K. Jin, C. Lu, H. Bei, B.C. Sales, L. Wang, L.K. Beland, R.E. Stoller, G.D. Samolyuk, M. Caro, A. Caro, W.J. Weber, Influence of chemical disorder on energy dissipation and defect evolution in concentrated solid solution alloys, Nat. Commun. 6 (2015) 8736.

[14] D.B. Miracle, O.N. Senkov, A critical review of high entropy alloys and related concepts, Acta Mater. 122 (Supplement C) (2017) 448-511.
[15] S.M. Liang, R. Schmid-Fetzer, Diffusion, evaluation of Calphad approach and empirical rules on the phase stability of multi-principal element alloys, J. Phase Equilib. Diffus. 38 (4) (2017) 369-381.

[16] C. Ng, S. Guo, J. Luan, Q. Wang, J. Lu, S. Shi, C.T. Liu, Phase stability and tensile properties of Co-free Al0.5CrCuFeNi2 high-entropy alloys, J. Alloy. Compd. 584 (2014) 530-537.

[17] Y.F. Ye, Q. Wang, J. Lu, C.T. Liu, Y. Yang, High-entropy alloy: challenges and prospects, Mater. Today 19 (6) (2016) 349-362.

[18] J.W. Yeh, S.K. Chen, S.J. Lin, J.Y. Gan, T.S. Chin, T.T. Shun, C.H. Tsau, S.Y. Chang, Nanostructured high-entropy alloys with multiple principal elements: novel alloy design concepts and outcomes, Adv. Eng. Mater. 6 (5) (2004) 299-303.

[19] Y. Zhang, Y.J. Zhou, J.P. Lin, G.L. Chen, P.K. Liaw, Solid-solution phase formation rules for multi-component alloys, Adv. Eng. Mater. 10 (6) (2008) 534-538.

[20] X. Yang, Y. Zhang, Prediction of high-entropy stabilized solid-solution in multicomponent alloys, Mater. Chem. Phys. 132 (2-3) (2012) 233-238.

[21] Y.F. Ye, C.T. Liu, Y. Yang, A geometric model for intrinsic residual strain and phase stability in high entropy alloys, Acta Mater. 94 (2015) 152-161.

[22] S. Guo, C. Ng, J. Lu, C.T. Liu, Effect of valence electron concentration on stability of fcc or bcc phase in high entropy alloys, J. Appl. Phys. 109 (10) (2011) 645-647.

[23] M.C. Troparevsky, J.R. Morris, P.R.C. Kent, A.R. Lupini, G.M. Stocks, Criteria for predicting the formation of single-phase high-entropy alloys, Phys. Rev. X 5 (2015) 011041.

[24] Z. Pei, J. Yin, J.A. Hawk, D.E. Alman, M.C. Gao, Machine-learning informed prediction of high-entropy solid solution formation: beyond the Hume-Rothery rules, NPJ Comput. Mater. 6 (2020) 50.

[25] W. Zhang, P.K. Liaw, Y. Zhang, Science and technology in high-entropy alloys, Sci. China Mater. 61 (1) (2018) 2-22.

[26] T. Stasiak, S.N. Kumaran, M. Touzin, F. Beclin, C. Cordier, novel multicomponent powders from the AlCrFeMnMo family synthesized by mechanical alloying, Adv. Eng. Mater. 21 (2019) 1900808.

[27] O.N. Senkov, G.B. Wilks, J.M. Scott, D.B. Miracle, Mechanical properties of Nb25Mo25Ta25W25 and V20Nb20Mo20Ta20W20 refractory high entropy alloys, Intermetallics 19 (2011) 698-706.

[28] M. Hillert, Phase Equilibria, Phase Diagrams and Phase Transformations: Their Thermodynamic Basis, 2nd ed., Cambridge University Press,, 2007, doi:10.1017/CBO9780511812781.

[29] L.J. Santodonato, P.K. Liaw, R.R. Unocic, H. Bei, J.R. Moris, Predictive multiphase evolution in Al-containing high-entropy alloys, Nat. Commun. 9 (2018) 4520.

[30] L.J. Zhang, K. Guo, H. Tang, M.D. Zhang, J.T. Fan, P. Gui, Y.M. Ma, P.F. Yu, G. $\mathrm{Li}$, The microstructure and mechanical properties of novel Al-Cr-Fe-Mn-Ni high-entropy alloys with trimodal distributions of coherent B2 precipitates, Mater. Sci. Eng. A 757 (2019) 160-171.

[31] E.T. Jaynes, Information theory and statistical mechanics, Phys. Rev. 106 (4) (1957) 620-630.

[32] M.C. Gao, M. Widom, Information entropy of liquid metals, J. Phys. Chem. B 122 (13) (2018) 3550-3555.

[33] R. Besson, J. Dequeker, L. Thuinet, A. Legris, Ab initio thermodynamics of complex alloys: the case of Al- and Mn-doped ferritic steels, Acta Mater. 169 (2019) 284-300. 